\title{
Low Proportion of Dietary Plant Protein among Athletes with Premenstrual Syndrome-Related Performance Impairment
}

\author{
Keiko Yamada ${ }^{1}$ and Takashi Takeda ${ }^{1}$ \\ ${ }^{1}$ Division of Women's Health, Research Institute of Traditional Asian Medicine, Kindai University, Osaka-sayama, \\ Osaka, Japan
}

\begin{abstract}
Premenstrual syndrome (PMS) is psychosomatic disorder that are limited to the late luteal phase in the menstrual cycle. PMS could impair athletic performance. To investigate associations between proportions of dietary plant and animal protein and PMS-related impairment of athletic performance, we surveyed 135 female athletes aged 18-23 years attending Kindai University. Participants belonged to authorized university clubs, all of which have high rankings in Japanese university sports. Participants completed selfadministered questionnaires on diet history, demographics, and PMS-related impairment of athletic performance. Total protein, animal protein, and plant protein intake were examined, and the proportion of dietary plant protein was calculated for each participant. We divided athletes into two groups: those without PMS-related impairment of athletic performance $(n=117)$ and those with PMS-related performance impairment $(n=18)$. A t-test was used to compare mean values and multivariable adjusted mean values between groups; adjustment variables were energy intake, body mass index, and daily training duration. Total protein intake was not significantly different between the groups. However, athletes whose performance was affected by PMS reported higher intake of animal protein (mean $50.6 \mathrm{~g}$ ) than athletes whose performance was unaffected by PMS (mean $34.9 \mathrm{~g}$ ). Plant protein intake was lower among athletes with PMS-related impairment (mean $25.4 \mathrm{~g}$ ) than among athletes without impairment (mean $26.9 \mathrm{~g}$ ). The proportion of dietary plant protein was lower among athletes with PMS-related impairment (39.3\%) than those without impairment (45.9\%). A low proportion of dietary plant protein may cause PMS-related athletic impairment among athletes.
\end{abstract}

Keywords: athletes; diet; performance; premenstrual syndrome; protein intake

Tohoku J. Exp. Med., 2018 February, 244 (2), 119-122. (C) 2018 Tohoku University Medical Press

\section{Introduction}

Premenstrual syndrome (PMS) is physical discomfort or dysphoria, including irritability, tension, depressed mood, tearfulness, and mood swings, that occurs in the weeks before menstruation and that is limited to the premenstrual phase, with recovery at the start of menstruation (Yonkers et al. 2008). Severe PMS that interferes with personal and social relationships is defined as premenstrual dysphoric disorder (PMDD) by the Diagnostic and Statistical Manual of Mental Disorders (DSM)-5 (American Psychiatric Association 2013). Among Japanese women of reproductive age, $1.2 \%$ has been diagnosed with PMDD and $5.3 \%$ with moderate-to-severe PMS (Takeda et al. 2006).

PMS can negatively affect the performance of athletes (Takeda et al. 2015); namely, managing PMS symptoms might improve their athletic performance. Changing dietary habits and lifestyle is considered the best non-pharmacological approach to improving PMS (Grady-Weliky
2003). However, there is insufficient evidence of the association between dietary habits and PMS. The aim of this study was to investigate the association between the relative proportions of dietary plant and animal protein and PMSrelated impairment of athletic performance among college athletes.

\section{Methods}

Study population and measures

In total, 141 female athletes aged 18-23 years attending Kindai University completed a brief self-administered diet history questionnaire (BDHQ) (Kobayashi et al. 2011) and a questionnaire about demographics and PMS-related impairment of athletic performance during training and competition (PSQ, Premenstrual Symptoms Questionnaire; Table 1) (Takeda et al. 2006). Data from six athletes who took hormonal therapy were excluded, leaving 135 questionnaires for analysis.

The BDHQ was a structured, self-administered questionnaire that included 58 food items for the assessment of diet history during the preceding month. The survey required approximately 15-20 minutes to complete. We calculated the relative proportion of dietary

Received December 19, 2017; revised and accepted January 25, 2018. Published online February 10, 2018; doi: 10.1620/tjem.244.119. Correspondence: Takashi Takeda, M.D., Ph.D., Division of Women's Health, Research Institute of Traditional Asian Medicine, Kindai

University, Osaka-sayama, Osaka 589-8511, Japan.

e-mail: take@med.kindai.ac.jp 
animal and plant protein, based on the food and beverage items included in the BDHQ (Kobayashi et al. 2011). Intake of total protein, animal protein, and plant protein were determined from the BDHQ, and the percentage of plant protein to total protein was calculated for each participant. We used the PSQ, which is a clinical PMS screening tool (Steiner et al. 2003; Takeda et al. 2006), to evaluate 11 PMS symptoms in each patient (Table 1). The PSQ translates DSM criteria into a rating scale, with degrees of severity described in Japanese; not at all, mildly, moderately, or severely. The athletes also indicated the degree to which these symptoms impaired their performance in training and competition as not at all, mildly, moderately, or severely. Athletes who reported moderate or severe impairment were categorized as having PMS-related performance impairment.

\section{Statistical analysis}

We divided athletes into two groups: those who reported PMSrelated performance impairment and those who did not. Means and multivariable-adjusted means were compared with a t-test; adjustment variables were energy intake, body mass index (BMI), and daily training duration. All statistical analyses were performed with SAS version 9.4 (SAS Institute Inc., Cary, NC, USA).

\section{Ethical approval}

This study was conducted in accordance with the Helsinki Declaration and was approved by the Kindai University Institutional Review Board. Informed consent was obtained from all individual participants included in the study.

\section{Results}

In total, 117 athletes $(86.7 \%)$ reported no PMS-related effects on performance and 18 athletes (13.3\%) reported PMS-related athletic impairment of performance. Among the 18 athletes with PMS-related athletic impairment, 11 athletes $(61.1 \%)$ were categorized as low degree of PMS severity, and 7 athletes (38.9\%) were as moderate to severe.

Mean values and proportions of sample characteristics according to group are shown in Table 2. Mean age and energy intake did not differ significantly between groups. Athletes whose performance was affected by PMS had higher average BMI $\left(24.1 \mathrm{~kg} / \mathrm{m}^{2}\right)$ than athletes unaffected by PMS $\left(21.7 \mathrm{~kg} / \mathrm{m}^{2}\right)$. The mean daily training time of athletes whose performance was affected by PMS was 172.8 minutes per day, whereas athletes unaffected by PMS trained 188.5 minutes per day. This difference was not statistically significant. Athletes whose performance was affected by PMS participated in the following sports: combat sports or martial arts $(7 / 18,38.9 \%)$, archery or Japanese art of archery $(4 / 18,22.2 \%)$, and other $(7 / 18,38.9 \%)$. Sports of the unaffected athletes included ball sports $(18 / 117,15.4 \%)$, athletic sports $(3 / 117,2.6 \%)$, combat sports or martial arts $(30 / 117,25.6 \%)$, archery or Japanese art of archery $(4 / 117,3.4 \%)$, other $(38 / 117,32.5 \%)$, and unanswered $(7 / 117,6.0 \%)$. The number of national-level athletes was $11 / 18(61.1 \%)$ in the former group and 59/117 $(50.4 \%)$ in the latter. This difference was not statistically significant. A higher proportion of athletes whose performance was affected by PMS (38.9\%) reported moderate-tosevere PMS than those without PMS-related impairment $(6.9 \%)$.

Multivariable adjusted mean values of protein intake by group are shown in Table 3. The adjusted mean age and energy intake did not differ significantly between groups: mean age, 19.8 years among athletes without PMS-related impairment vs. 20.1 years among those with impairment; and energy intake, 1,768.9 kcal among athletes without

Table 1. Premenstrual Symptoms Questionnaire (PSQ), a clinical premenstrual syndrome screening tool.

Q1 Within the last three months, have you experienced the following premenstrual symptoms
starting the week before menses and ending within a few days after the onset of menses?
1 Depressed mood
2 Anxiety or tension
3 Tearfulness
4 Anger or irritability
5 Decreased interest in work, home, or social activities
6 Difficulty concentrating
7 Fatigue or lack of energy
8 Overeating or food cravings
9 Insomnia or hypersomnia
10 Feeling overwhelmed
11 Physical symptoms such as tender breasts, feeling bloated, headache, joint or muscle
pain, or weight gain

Q2 How much have these symptoms impaired your performance in training and competition?

Not at all Mildly Moderately Severely


Table 2. Characteristics of participants according to effect of PMS on athletic performance.

\begin{tabular}{lll}
\hline & $\begin{array}{l}\text { Athletes without PMS-related } \\
\text { performance impairment }\end{array}$ & $\begin{array}{l}\text { Athletes with PMS-related performance } \\
\text { impairment }\end{array}$ \\
\hline $\mathrm{n}$ & 117 & 18 \\
Age, years (SE) & $19.8(0.1)$ & $20.1(0.3)$ \\
Energy intake, $\mathrm{kcal}(\mathrm{SE})$ & $1768.9(67.7)$ & $1870.9(172.7)$ \\
Body mass index, $\mathrm{kg} / \mathrm{m}^{2}(\mathrm{SE})$ & $21.7(0.3)$ & $24.1(0.7)^{\dagger}$ \\
Daily training time, minutes (SE) & $188.5(8.0)$ & $172.8(20.5)$ \\
& & \\
Kind of sport, $\mathrm{n}(\%)$ & & 0 \\
$\quad$ Ball sports & $18(15.4 \%)$ & 0 \\
$\quad$ Athletic sports & $3(2.6 \%)$ & $7(38.9 \%)$ \\
Combat sports or martial arts & $30(25.6 \%)$ & $4(22.2 \%)$ \\
Archery or Japanese art of archery & $4(3.4 \%)$ & $7(38.9 \%)$ \\
Other & $38(32.5 \%)$ & 0 \\
$\quad$ Unanswered & $7(6.0 \%)$ & $11(61.1 \%)$ \\
Number of national level athletes, $\mathrm{n}(\%)$ & $59(50.4 \%)$ & $38.9 \% 0^{\ddagger}$ \\
\hline
\end{tabular}

† $<0.01,{ }^{\star} \mathrm{p}<0.001$ for intergroup comparison.

PMS, premenstrual syndrome; SE, standard error.

Table 3. Multivariable adjusted mean values of protein intake according to effect of PMS on athletic performance.

\begin{tabular}{lll}
\hline & $\begin{array}{l}\text { Athletes without PMS-related performance } \\
\text { impairment }\end{array}$ & $\begin{array}{l}\text { Athletes with PMS-related performance } \\
\text { impairment }\end{array}$ \\
\hline $\mathrm{n}$ & 117 & 18 \\
Total protein intake, g (SE) & $61.9(1.9)$ & $76.0(5.0)$ \\
Animal protein intake, $\mathrm{g}(\mathrm{SE})$ & $34.9(2.1)$ & $50.6(5.5)^{*}$ \\
Plant protein intake, $\mathrm{g}$ (SE) & $26.9(0.5)$ & $25.4(1.2)^{*}$ \\
Plant protein percentage of total protein intake & $45.9 \%$ & $39.3 \%^{*}$ \\
\hline
\end{tabular}

Adjusted for energy intake, body mass index, and daily training time.

$* p<0.05$ for intergroup comparison.

PMS, premenstrual syndrome; SE, standard error.

PMS-related impairment vs. $1,870.9 \mathrm{kcal}$ among those with impairment. Total protein intake also did not differ significantly between groups; however, mean animal protein intake was higher among athletes with PMS-related impairment $(50.6 \mathrm{~g})$ than among those without impairment (34.9 g). Mean intake of plant protein was lower among athletes with PMS-related impairment $(25.4 \mathrm{~g})$ than among those without impairment $(26.9 \mathrm{~g})$. The proportion of plant protein was also lower among athletes with impairment $(39.3 \%)$ than among those without impairment (45.9\%).

\section{Discussion}

As reported in a previous study (Takeda et al. 2015), we found that a sizable percentage of participants $(13 \%, 18$ of 135 athletes) reported that PMS had negative effects on their performance during training and competition. A large proportion of athletes $(61.1 \%, 11$ of 18 athletes) who reported PMS-related athletic impairment showed low PMS degree. PMS-related athletic impairment may be different from PMS-related daily life impairment, and a proportion of plant protein may affect only sports performance because improvement of sports performance by plant protein intake was described in the previous study (Ren et al. 2017).

A low proportion of dietary plant protein may cause PMS-related athletic impairment in female athletes. This hypothesis is consistent with the findings of a previous study, which reported that soy isoflavones prevented PMS symptoms among Korean women living in the U.S. (Kim et al. 2006). Although we could not analyze the components of the plant proteins consumed because of the small sample size in the current study, Asian populations are traditionally heavy consumers of soy products (e.g., soy sauce, soybean paste, tofu).

Athletes often consume high levels of animal protein in the belief that this will lead to better performance. However, a low proportion of dietary plant protein may cause PMS-related athletic impairment in athletes.

The present study had some limitations. First, the 
respondents may not be truly representative of female athletes in general. Second, the cross-sectional design of the study cannot indicate a clear direction of causality. Prospective studies on the association among nutrition, dietary habits, and PMS should be performed to reveal causality. Third, the physical demands of training may have been different between groups in this study, which could have affected the severity of PMS. However, we could not stratify participants according to sport because of the small sample size.

These limitations notwithstanding, our results suggest that a low proportion of dietary plant protein may cause PMS-related athletic impairment in female athletes.

\section{Acknowledgments}

This work was supported by JSPS KAKENHI grant number 15K01636, Tokyo, Japan and Research Promotion and Practical Use for Women's Health, AMED grant number 15666492, Tokyo, Japan.

We thank Bonnie Lynch, Ph.D., and Rebecca Tollefson, D.V.M., from Edanz Group (https://www.edanzediting.com/ac) for editing drafts of this manuscript.

\section{Conflict of Interest}

The authors declare no conflict of interest.

\section{References}

American Psychiatric Association (2013) Diagnostic and Statis- tical Manual of Mental Disorders, 5th ed. (DSM-5), American Psychiatric Press, Washington, DC.

Grady-Weliky, T.A. (2003) Clinical practice. Premenstrual dysphoric disorder. N. Engl. J. Med., 348, 433-438.

Kim, H.W., Kwon, M.K., Kim, N.S. \& Reame, N.E. (2006) Intake of dietary soy isoflavones in relation to perimenstrual symptoms of Korean women living in the USA. Nurs. Health Sci., 8, 108-113.

Kobayashi, S., Murakami, K., Sasaki, S., Okubo, H., Hirota, N., Notsu, A., Fukui, M. \& Date, C. (2011) Comparison of relative validity of food group intakes estimated by comprehensive and brief-type self-administered diet history questionnaires against $16 \mathrm{~d}$ dietary records in Japanese adults. Public Health Nutr., 14, 1200-1211.

Ren, G., Yi, S., Zhang, H. \& Wang, J. (2017) Ingestion of soywhey blended protein augments sports performance and ameliorates exercise-induced fatigue in a rat exercise model. Food Funct., 8, 670-679.

Steiner, M., Macdougall, M. \& Brown, E. (2003) The premenstrual symptoms screening tool (PSST) for clinicians. Arch. Womens Ment. Health, 6, 203-209.

Takeda, T., Imoto, Y., Nagasawa, H., Muroya, M. \& Shiina, M. (2015) Premenstrual syndrome and premenstrual dysphoric disorder in Japanese collegiate athletes. J. Pediatr. Adolesc. Gynecol., 28, 215-218.

Takeda, T., Tasaka, K., Sakata, M. \& Murata, Y. (2006) Prevalence of premenstrual syndrome and premenstrual dysphoric disorder in Japanese women. Arch. Womens Ment. Health, 9, 209-212.

Yonkers, K.A., O’Brien, P.M.S. \& Eriksson E. (2008) Premenstrual syndrome. Lancet, 371, 1200-1210. 